\title{
The use of laryngeal mask airway in dental treatment during sevoflurane deep sedation
}

\author{
Sangeun Lee', Jongsoo Kim', Jongbin Kim', Seungoh $\mathrm{Kim}^{2}$ \\ 'Department of Pediatric Dentistry, School of Dentistry, Dankook University, ${ }^{2}$ Department of Anesthesiology, School of Dentistry, Dankook \\ University, Cheonan, Korea
}

\begin{abstract}
Background: General anesthesia is frequently considered for pediatric patients, as they often find it difficult to cooperate and stay calm during administration of potentially painful treatments. Sedation can overcome these adversities; however, this is challenging while maintaining unobstructed airways.

Methods: The study involved 11 pediatric dental patients treated with LMA under deep sedation with sevoflurane, from 2011 through 2015. LMA size, sevoflurane concentration, and the vital signs of patients were assessed through a chart review.

Results: The age distribution of the patients ranged from 6 to 10 years old. A total of 3 patients underwent mesiodens extraction, while the remaining 8 underwent an surgically assisted orthodontic forced tooth eruption The average sedation period was approximately 45 minutes and the LMA size was $2 \frac{1}{2}$. The sevoflurane concentration was maintained at $2 \%$ on average, and overall, the measurements of vital signs were within the normal range; the patients had an average blood pressure of $98 / 49 \mathrm{mmHg}$, breathing rate of 26 times/min, pulse frequency of 95 times/min, $\mathrm{SpO}_{2} \mathrm{~s}$ level of $99 \mathrm{mmHg}$, and $\mathrm{ETCO}_{2}$ level of $41.2 \mathrm{mmHg}$.

Conclusions: Deep sedation with sevoflurane coupled with LMA may be applied successfully in pediatric patients who undergo mesiodens extraction or a surgically assisted orthodontic forced tooth eruption
\end{abstract}

Key Words: Dental treatment; Deep sedation; Laryngeal mask airway; Sevoflurane.

\section{INTRODUCTION}

In pediatric dentistry, mesiodens extraction or surgically assisted orthodontic forced tooth eruption (window opening to induce natural eruption of teeth) is an invasive procedure that requires the precise handling of dental equipment. Therefore, the safe and successful completion of these procedures requires cooperation from patients. General anesthesia is an option for pediatric patients undergoing dental procedures. However, both patients and parents may feel pressure due to the considerable length of time required for the maintenance of venous access and endotracheal intubation, in addition to a prolonged recovery period. Another potential disadvan- tage to general anesthesia may be the post-procedural discomfort surrounding the throat.

On the other hand, sevoflurane deep sedation allows the maintenance of spontaneous respiration in patients. Endotracheal intubation or the injection of a muscle relaxant is unnecessary, and therefore, the overall duration of anesthesia can be shortened. Moreover, the degree of sedation can be easily manipulated by altering the concentration of sevoflurane, allowing appropriate behavioral management of the patient for the dental treatment. However, the use of a saline solution in cases of bleeding or general washing during the treatment may cause difficulties in the maintenance of spontaneous respiration for patients. LMA can be helpful to resolve this issue by facilitating unobstructed airways. Moreover, LMA
Copyright(c) 2016 Journal of Dental Anesthesia and Pain Medicine
Received: 2016. March. 10. • Revised: 2016. March. 29. - Accepted: 2016. March. 31.

Corresponding Author: Seungoh Kim, Department of Anesthesiology, School of Dentistry, Dankook University, 119

Dandaero, Dongnam-gu, Cheonan, 31116, Korea

Tel: +82-41-550-1863 Fax: +82-41-550-1863 E-mail: ksomd@naver.com 
enables positive pressure ventilation in cases of apnea by forming a low-pressure seal around the laryngeal inlet.

This study examines the effectiveness of deep sedation with LMA in minor oral surgeries by measuring physiological parameters including the breathing patterns of patients.

\section{MATERIALS AND METHODS}

\section{Subjects}

With permission from Dankook University Dental Hospital's institutional review board (IRB), this study focused on 11 pediatric dental patients who had undergone dental treatment using LMA under deep sedation with sevoflurane from 2011 to 2015 .

Written consent was obtained prior to sedation from all patients, and deep sedation with sevoflurane was induced using LMA after an 8 hour fasting period (Fig.

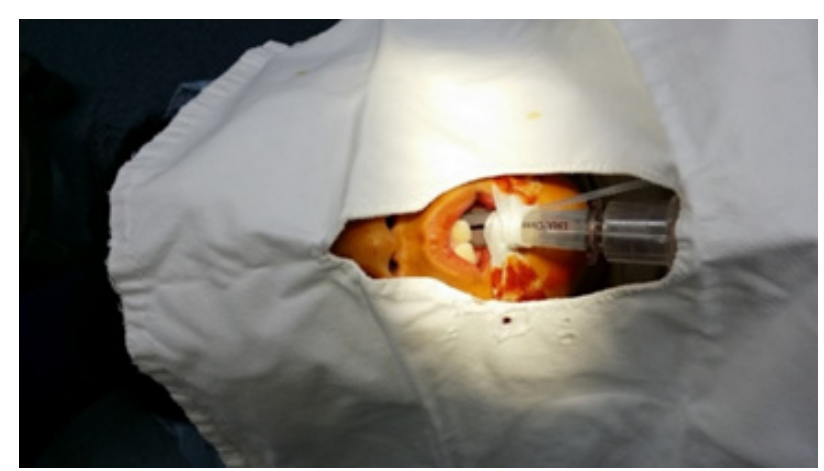

Fig. 1. LMA insertion for deep sevoflurane sedation, used for dental treatment.
1). To ensure the patient's safety, breathing rate, pulse rate, blood pressure, $\mathrm{SpO}_{2}$, and $\mathrm{ETCO}_{2}$ levels were observed and recorded.

\section{Methods}

Through a chart review, the basic characteristics of the patients, treatments performed, the duration of sedation, and LMA sizes were analyzed. In addition, the concentration of sevoflurane inhaled during the treatment, breathing and pulse rates, blood pressure, and $\mathrm{SpO}_{2}$ and $\mathrm{ETCO}_{2}$ levels were analyzed; minimum, maximum, and average values were calculated. This study followed the categorical normal ranges obtained from the standards of the 5th edition of "Pediatric and adolescence dentistry" [1]. Standard readings for the highest and lowest blood pressures in school-aged patients were $90-135 \mathrm{mmHg}$ and 49-70 $\mathrm{mmHg}$, respectively. Other standard measurements such as breathing and pulse rates, $\mathrm{SpO}_{2}$ and $\mathrm{ETCO}_{2}$ levels ranged from 18-30 times/min, 70-120 beats/min, $\geq 95$ $\mathrm{mmHg}$, and $23-51 \mathrm{mmHg}$ respectively.

\section{RESULTS}

Most patients were elementary school-aged, with an age distribution range from 6 to 10 years (mean age 8.1 $\pm 1.4)$. According to age, the weight distribution ranged from 18 to $53 \mathrm{~kg}$, (mean weight $33 \pm 13 \mathrm{~kg}$ ) (Table 1). Mesiodens extraction was performed in 3 patients, and the orthodontic forced tooth eruption (window opening to induce the natural eruption of permanent teeth) was

Table 1. Patient information, dates of visits, and treatment methods

\begin{tabular}{cccccc}
\hline Name & Date of visit & Age & Gender & Weight $(\mathrm{kg})$ & Treatment \\
\hline Kim00 & $2011-11-28$ & 8 & $\mathrm{M}$ & 53 & W/0 \\
Kim00 & $2013-11-19$ & 8 & $\mathrm{M}$ & 53 & W/0 \\
Gil00 & $2014-03-18$ & 8 & $\mathrm{~F}$ & 30 & Mesiodens ext. \\
Park00 & $2014-05-13$ & 6 & $\mathrm{~F}$ & 18 & Mesiodens ext. \\
Lee00 & $2014-05-21$ & 7 & $\mathrm{~F}$ & 25 & Mesiodens ext. \\
Ch000 & $2014-05-27$ & 6 & $\mathrm{~F}$ & 21 & W/0 \\
Lim00 & $2014-08-28$ & 8 & $\mathrm{~F}$ & 21 & W/0 \\
Kim00 & $2014-09-23$ & 10 & $\mathrm{M}$ & 53 & W/0 \\
Kim00 & $2014-11-06$ & 8 & $\mathrm{~F}$ & $\mathrm{~W}$ & W/0 \\
Ch000 & $2015-02-25$ & 10 & $\mathrm{~F}$ & 39.3 & W/0 \\
Park00 & $2015-03-11$ & 10 & 30 & W/0 \\
\hline
\end{tabular}

F: female, M: male, W/O: window opening, ext: extraction. 
Table 2. Blood pressure and breathing rates of the patients

\begin{tabular}{|c|c|c|c|c|c|c|c|}
\hline \multirow{2}{*}{ Age } & \multirow{2}{*}{ Gender } & \multicolumn{3}{|c|}{ Blood pressure } & \multicolumn{3}{|c|}{ Breathing rates } \\
\hline & & $\max$ & $\min$ & avg & $\max$ & $\min$ & avg \\
\hline 6 & $\mathrm{~F}$ & $128 / 63$ & $100 / 43$ & $109 / 48.4$ & 35 & 26 & 30.8 \\
\hline 6 & $\mathrm{~F}$ & $100 / 65$ & $71 / 35$ & $82.5 / 42$ & 42 & 29 & 35.4 \\
\hline 7 & $\mathrm{~F}$ & $103 / 53$ & $89 / 45$ & $93.8 / 48.5$ & 24 & 15 & 19.4 \\
\hline 8 & $M$ & $132 / 75$ & $93 / 43$ & $107 / 59$ & 31 & 24 & 27.4 \\
\hline 8 & M & $135 / 68$ & $112 / 48$ & $124 / 48$ & 31 & 16 & 26.3 \\
\hline 8 & $\mathrm{~F}$ & $119 / 71$ & $90 / 52$ & $101.5 / 57$ & 32 & 18 & 28.2 \\
\hline 8 & $\mathrm{~F}$ & $106 / 65$ & $85 / 40$ & $89.9 / 49.5$ & 19 & 14 & 16.6 \\
\hline 8 & $\mathrm{~F}$ & $105 / 60$ & $80 / 59$ & $86.1 / 44.5$ & 39 & 29 & 34.8 \\
\hline 10 & M & $135 / 66$ & $90 / 40$ & $98 / 45$ & 30 & 26 & 27.6 \\
\hline 10 & M & $113 / 62$ & $86 / 43$ & $90 / 47$ & 22 & 18 & 20.3 \\
\hline 10 & $\mathrm{~F}$ & $145 / 97$ & $75 / 35$ & $91 / 50$ & 24 & 18 & 22.7 \\
\hline
\end{tabular}

max: maximum, min: minimum, avg: average, $\mathrm{F}$ : female, $\mathrm{M}$ : male

Table 3. Pulse rates, $\mathrm{SpO}_{2}$ and $\mathrm{ETCO}_{2}$ levels of the patients

\begin{tabular}{|c|c|c|c|c|c|c|c|c|}
\hline \multirow{2}{*}{ Age } & \multirow{2}{*}{ Gender } & \multicolumn{3}{|c|}{ Pulse rate } & \multicolumn{2}{|c|}{$\mathrm{SpO}_{2}$} & \multicolumn{2}{|c|}{$\mathrm{ETCO}_{2}$} \\
\hline & & Max & $\min$ & avg & $\max$ & $\min$ & $\max$ & $\min$ \\
\hline 6 & $\mathrm{~F}$ & 120 & 90 & 99.2 & 100 & 99 & 59 & 28 \\
\hline 6 & $\mathrm{~F}$ & 115 & 85 & 87.9 & 100 & 100 & 43 & 27 \\
\hline 7 & $\mathrm{~F}$ & 118 & 89 & 93.5 & 99 & 99 & 39 & 26 \\
\hline 8 & $M$ & 130 & 87 & 108 & 99 & 99 & 64 & 50 \\
\hline 8 & $M$ & 118 & 81 & 108 & 100 & 100 & 63 & 26 \\
\hline 8 & $\mathrm{~F}$ & 105 & 82 & 92.5 & 100 & 99 & 49 & 35 \\
\hline 8 & $\mathrm{~F}$ & 103 & 65 & 83 & 99 & 99 & 30 & 28 \\
\hline 8 & $\mathrm{~F}$ & 128 & 89 & 98.1 & 100 & 99 & 43 & 38 \\
\hline 10 & $\mathrm{M}$ & 142 & 85 & 104 & 98 & 97 & 58 & 50 \\
\hline 10 & $M$ & 87 & 70 & 77 & 100 & 100 & 45 & 34 \\
\hline 10 & $\mathrm{~F}$ & 160 & 75 & 100 & 100 & 98 & 43 & 28 \\
\hline
\end{tabular}

max: maximum, min: minimum, avg: average, F: female, M: male

performed in the remaining 8 patients. All 3 mesiodens extraction patients were female, and the group of 8 patients who underwent the window opening procedure included 4 male and 4 female patients. No other underlying or systemic diseases were present in any patients.

The average sedation period was 45 minutes, and the median size of LMA used was $2 \frac{1}{2}$. Sevoflurane concentration was at $4 \%$ for the initial induction stage, and was maintained at $2 \%$ for the remaining treatment period. All patients maintained deep sedation and were unconscious.

The average blood pressure and breathing rates were 98/49 $\mathrm{mmHg}$ and 26 times/min. Minimum, maximum, and average values for individual patients are outlined in Table 2. Average pulse rates, $\mathrm{SpO}_{2}$ and $\mathrm{ETCO}_{2}$ levels were 95 beats/min, $99 \mathrm{mmHg}$, and $41.2 \mathrm{mmHg}$, respec- tively. Minimum, maximum, and average values for individual patients are outlined in Table 3 .

\section{DISCUSSION}

Sevoflurane is a volatile inhalation anesthetic, discovered by two scientists - Ross Terrell and Bernard M. Regan [2]. The discovery and detailed characterization of sevoflurane were first shown in the paper by Wallin et al., 1975 [3]. Sevoflurane was first implemented in clinical settings in Japan in the 1990s.

Deep sedation using sevoflurane was proven to be a safe and effective method for dental treatments, with statistical significance, in two separate studies by Lahoud et al. $[4,5]$. Sevoflurane coupled with $\mathrm{N}_{2} \mathrm{O}$ demonstrated higher success rates for dental treatments, faster recovery 
time, and reduced side-effects in comparison to cases that used $\mathrm{N}_{2} \mathrm{O}$ alone. The study by Paris et al. compared sedation results between 100 pediatric patients using $\mathrm{N}_{2} \mathrm{O}$, halothane, and sevoflurane. Significant results from this study indicated shorter induction periods and lower chances of cardiac arrhythmia in sevoflurane-sedated patients compared to halothane-sedated patients [6].

In the study by Lee et al., the authors mentioned that the use of sevoflurane can be an effective alternative to general anesthesia in pediatric dental patients [7]. Supporting evidence indicated the average induction time for sedation was 95 seconds; the average time from the end of sedation, until the patient can open their eyes, was 8 minutes; and the average time until complete recovery occurred was 30 minutes. Therefore, sevoflurane sedation is considered an effective method for pediatric patients with apprehension to dental procedures or uncontrolled behavioral management.

LMA was first discovered in 1981 by a doctor of the Royal London Hospital. Dr. Archie Brain, a British anesthesiologist, improved the Goldman Dental Mask so that it was positioned near the laryngeal inlet instead of the nasal cavity [8].

Unlike the nasal cannula, LMA can minimize gas leakage, allow the maintenance of high level sedation, and provide greater levels of comfort during dental procedures. Moreover, airways are less restricted with LMA in comparison to the nasal cannula. According to the study by Brimacombe and Berry, there are four requirements for the provision of a stable: (1) unobstructed airway, (2) the protection of lungs from aspiration, (3) minimal interference with the visual field for surgery, and (4) low complication rates [9]. Neither endotracheal intubation nor the nasal mask meets the above requirements; LMA provides an alternative with more benefits than risks. The benefits of LMA included an insertion without the use of a laryngoscope or muscle relaxants, and an air-tight seal around the laryngeal inlet. Consequently, the induction time for anesthesia was reduced, and the airways were secured for spontaneous or controlled ventilation. The scavenging of waste gases is possible and is well tolerated by patients during recovery, shortening overall recovery time in patients with short, minor oral surgeries [10].

In addition, the study by $\mathrm{Yu}$ and Beirne showed significantly lower rates of laryngospasm and improved neck conditions in patients that used LMA as opposed to endotracheal tubes under general anesthesia [11]. The study by Bennett et al. also demonstrated lower rates of laryngospasm, reduced oxygen saturation levels, lower levels of laryngopharyngeal pain, and less coughing during the recovery in patients treated via LMA as opposed to endotracheal tubes [12]. This was due to the large diameter of LMA, which resulted in reduced resistance from the airways. Therefore, spontaneous respiration was easier, and the degree of airway security was comparable to endotracheal intubation but showed lower complication rates. Since the patient was under spontaneous respiration, there was a risk for respiratory acidosis as $\mathrm{ETCO}_{2}$ levels increase with reduced respiration volume. However, in most cases, respiratory acidosis did not induce any severe problems.

Typically, LMA allows lighter anesthesia and faster recovery, and is easier to implement than endotracheal intubation. According to the study by Morse et al., short and simple training is sufficient to provide the fast and effective insertion of LMA. The importance is emphasized in cases which endotracheal intubation is not feasible [13].

Throughout the treatment procedures performed in this study, all physiological parameters were stable within the normal hemodynamic ranges for all 11 patients who underwent dental treatment under deep sevoflurane sedation using LMA. In addition, sedation was stably maintained throughout the entire procedure in all patients.

\section{CONCLUSIONS}

Deep sedation with sevoflurane is a more effective, practical, and safe method in comparison to other sedation methods that use $\mathrm{N}_{2} \mathrm{O}$ or a combination of $\mathrm{N}_{2} \mathrm{O}$ and 
halothane. The rate of successful treatments was higher, in addition to faster recovery rates and the reduction of side-effects. Therefore, sevoflurane sedation may be an effective alternative to general anesthesia.

LMA may minimize gas leakage, maintain high levels of sedation, and provide comfort for patients with easier airway securing. In addition, in comparison to endotracheal intubation, LMA has lower complication rates and maintains the same degree of airway security. The effectiveness of dental treatments is also improved, as LMA allows is conducive to spontaneous respiration in patients under deep sedation. Lastly, both short anesthetic induction times and reduced recovery periods make this method very useful for emergency dental treatments. In conclusion, LMA can be successfully used during deep sedation with sevoflurane for pediatric patients undergoing minor oral surgeries, such as mesiodens extraction or surgically assisted orthodontic forced tooth eruption.

\section{REFERENCES}

1. Korean Academy of Pediatric Dentistry. Pediatric Dentistry. 5th ed. Seoul, Dental Wisdom. 2014.

2. Terrell RC. The invention and development of enflurane, isoflurane, sevoflurane, and desflurane. Anesthesiology 2008; 108: 531-3.

3. Wallin RF, Regan BM, Napoli MD, Stern IJ. Sevoflurane: a new inhalational anesthetic agent. Anesth Analg 1975; 54: 758-66

4. Lahoud G, Averley P, Hanlon M. Sevoflurane inhalation conscious sedation for children having dental treatment.
Anaesthesia 2001; 56: 476-80.

5. Lahoud G, Averley P. Comparison of sevoflurane and nitrous oxide mixture with nitrous oxide alone for inhalation conscious sedation in children having dental treatment: a randomised controlled trial. Anaesthesia 2002; 57: 446-50.

6. Paris S, Cafferkey M, Tarling M, Hancock P, Yate P, Flynn P. Comparison of sevoflurane and halothane for outpatient dental anaesthesia in children. Br J Anaesth 1997; 79: 280-4.

7. Lee M, Bennett HE, Gordon N. Sevoflurane general anesthesia: an alternative technique in the pediatric oral and maxillofacial surgery patient. J Oral Maxillofac Surg 2003; 61: 1249-52.

8. Chmielewski C, Snyder-Clickett S. The use of the laryngeal mask airway with mechanical positive pressure ventilation. AANA J 2004; 72: 347-51.

9. Brimacombe J, Berry A. The laryngeal mask airway for dental surgery a review. Aust Dent J 1995; 40: 10-4.

10. Goodwin A, Ogg T, Lamb W, Adlam D. The reinforced laryngeal mask in dental day surgery. Ambul Surg 1993; 1: 31-5.

11. Seung HY, Beirne OR. Laryngeal mask airways have a lower risk of airway complications compared with endotracheal intubation: a systematic review. J Oral Maxillofac Surg 2010; 68: 2359-76.

12. Bennett J, Petito A, Zandsberg S. Use of the laryngeal mask airway in oral and maxillofacial surgery. J Oral Maxillofac Surg 1996; 54: 1346-51.

13. Morse Z, Sano K, Kageyama I, Kanri T. The relationship of placement accuracy and insertion times for the laryngeal mask airway to the training of inexperienced dental students. Anesth Prog 2002; 49: 9. 